\title{
Waiting for Godot: the Failure of SMEs in the Italian Manufacturing Industry to Grow
}

\author{
Luca Bagnato \\ Eleonora Bartoloni \\ Maurizio Baussola
}

Quaderno n. 132/marzo 2018 


\title{
DIPARTIMENTO DI SCIENZE ECONOMICHE E SOCIALI
}

\section{Waiting for Godot: the Failure of SMEs in the Italian Manufacturing Industry to Grow}

\author{
Luca Bagnato* \\ Eleonora Bartoloni* \\ Maurizio Baussola*
}

Quaderno n. 132/marzo 2018

\section{VP VITA E PENSIERO}

- UCSC, Università Cattolica del Sacro Cuore, Dipartimento di Scienze Economiche e Sociali, Via Emilia Parmense 12, Piacenza 29100, Italy. E-mail: luca.bagnato@unicatt.it.

* ISTAT, Italian National Institute of Statistics, Via Oldofredi 23, Milano 20124 and Università di Parma, Dipartimento di Scienze Economiche e Aziendali, Via J.F. Kennedy 6, Parma 43125, Italy. E-mail: bartolon@istat.it.

- UCSC, Università Cattolica del Sacro Cuore, Dipartimento di Scienze Economiche e Sociali, Via Emilia Parmense 12, Piacenza 29100, Italy. E-mail: maurizio.baussola@unicatt.it. 
Luca Bagnato, Dipartimento di Scienze Economiche e Sociali, Università Cattolica del Sacro Cuore, Piacenza.

Eleonora Bartoloni, ISTAT, Italian National Institute of Statistics, via Oldofredi, 23 - 20124 Milano and Università di Parma, Dipartimento di Scienze Economiche e Aziendali, via J.F. Kennedy 6 - 43125 Parma.

Maurizio Baussola, Dipartimento di Scienze Economiche e Sociali, Università Cattolica del Sacro Cuore, Piacenza.

$\triangle 1$ luca.bagnato@unicatt.it

$\triangle$ bartolon@istat.it

$\bowtie$ maurizio.baussola@unicatt.it

I quaderni possono essere richiesti a:

Dipartimento di Scienze Economiche e Sociali, Università Cattolica del Sacro Cuore

Via Emilia Parmense 84 - 29122 Piacenza - Tel. 0523599.342

http://dipartimenti.unicatt.it/dises

\section{$\bowtie$ dises-pc@unicatt.it}

www.vitaepensiero.it

All rights reserved. Photocopies for personal use of the reader, not exceeding $15 \%$ of each volume, may be made under the payment of a copying fee to the SIAE, in accordance with the provisions of the law n. 633 of 22 april 1941 (art. 68, par. 4 and 5). Reproductions which are not intended for personal use may be only made with the written permission of CLEARedi, Centro Licenze e Autorizzazioni per le Riproduzioni Editoriali, Corso di Porta Romana 108, 20122 Milano, e-mail: autorizzazioni@clearedi.org,web site www.clearedi.org.

Le fotocopie per uso personale del lettore possono essere effettuate nei limiti del 15\% di ciascun volume dietro pagamento alla SIAE del compenso previsto dall'art. 68, commi 4 e 5, della legge 22 aprile 1941 n. 633.

Le fotocopie effettuate per finalità di carattere professionale, economico o commerciale o comunque per uso diverso da quello personale possono essere effettuate a seguito di specifica autorizzazione rilasciata da CLEARedi, Centro Licenze e Autorizzazioni per le Riproduzioni Editoriali, Corso di Porta Romana 108, 20122 Milano, e-mail: autorizzazioni@clearedi.org e sito web www.clearedi.org.

(C) 2018 Luca Bagnato, Eleonora Bartoloni, Maurizio Baussola ISBN 978-88-343-3589-5 


\begin{abstract}
We use a panel of Italian manufacturing firms for the period 2001-2014 to analyse the distribution of firm size, and then test for the validity of Gibrat's law using unit root tests. Although Gibrat's Law is rejected and the estimates suggest that small firms grow faster than larger ones, we do not observe a significant change in the average size of companies at the end of the period under investigation. Also, by using a long-run Transition Probability Matrix, we verify that the steady-state distribution of firm size remains stable. The higher propensity to grow shown by smaller firms is confined to the size class in which the firm is established. We further investigate the relationship between the rate of growth in a firm's size conditional on specific firm and industry characteristics. Export intensity plays a significant role in affecting the size growth rate together with industry characteristics related to technological levels. Finally, we estimate the probability that a firm increases in size relative to the mean size prevailing in its own size class over a 14-year interval. This approach enables us to highlight those factors that affect this probability, thereby enabling us to underline how Gibrat's Law tests, although important, require complementary analysis to ascertain whether a firm's propensity to increase in size is a long run effect and thus a significant modification of the distribution of company size or only implies a marginal increase in size within a reference size class.
\end{abstract}

JEL Classifications: L11, L2, L6

Keywords: Gibrat's Law, Lognormal distribution, firm size distribution. 



\section{Introduction}

The analysis of firm size distribution has long been considered within the debate concerning the ability of small and medium-size enterprises to grow at the international level.

Typically this literature has focused on tests on observance of Gibrat's Law, i.e. that a firm's growth is independent of its initial size. The literature on this topic has increased significantly over the years; we aim to focus on those issues that still need to be investigated in more detail. In particular, we want to emphasise how tests for the validity of Gibrat's Law do not respond to the initial issue, i.e. whether small and mediumsize firms grow significantly more than their larger counterparts, and thus cause a change in the long-run distribution of firms. The results of these tests may suggest, for example, that Gibrat's Law does not hold and that small firms grow faster than larger firms. However, this contribution may be negligible and may not affect the long run distribution of firm size. Much of the literature has focused on the most appropriate methodology for testing validity of Gibrat's Law, and less attention has been paid to the long-run effect of the estimated results, which crucially entails considering the ability to grow of small and medium size enterprises in comparison with large companies. The seminal studies by ?? define the methodological framework in which proper analyses and tests have since been developed. In particular, more recent studies have focused on the need to consider tests of Gibrat's Law in a dynamic panel data framework. 
Goddard et al. (2002); Chen and Lu (2003); Oliveira and Fortunato (2006b) apply dynamic panel data estimation techniques and the panel unit root test to test Gibrat's Law for Japanese, Taiwanese and Portuguese data sets. This literature is closely related to the investigations dealing with panel unit root tests (Im et al., 2003; Choi, 2001; Levin et al., 2002) which have transferred previous results of time series unit root tests to a panel framework. In addition, other studies have highlighted the role of industry-specific effects, age and the birth or death rate of firms (Almus, 2000; Lotti et al., 2009; Fotopoulos and Giotopoulos, 2010). Our research falls within this stream of investigation and our aim is to test whether the growth rate of SMEs does significantly contribute to a modification of size distribution of firms in the longrun. Additionally, we estimate those factors that contribute to such a growth path. We use a large and representative data set of Italian manufacturing firms for the period 2001-2014, which enables us to derive significant and robust results. The size distribution of Italian firms has always been much discussed, and this debate is crucial because the ability of firms to grow ultimately offers more opportunities for a country's long-run growth. Technological and non-technological innovation, together with all kinds of innovation involving managerial and administrative approaches, may be better introduced and exploited if businesses evolve towards more structured organizations with the ability to interact in a globally competitive market. For these reasons it is relevant to investigate whether such a pattern is in place, and what factors may 
potentially drive the growth rate of firms. The paper is organised as follows. Section 2 discusses the main findings on firms' growth rate in the light of the empirical evidence prevailing internationally. Section 3 presents the data used for the empirical investigation and discusses the main stylised facts concerning firms' growth rate in the Italian manufacturing industry. Section 4 presents the tests for Gibrat's Law and the analysis of those firm-specific factors that crucially affect firms' growth rate. Given this investigation, we specify in Section 5 a long-run logit model that enables us to ascertain the long-term effect of those factors that have a positive effect on the probability of increasing a firm's size relative to the mean size class. Section 6 concludes the paper.

\section{Firms' growth: the analytical framework}

The literature on firms' growth has typically focused on the more appropriate methodology to test for the so called Gibrat's Law, according to which a firm's growth rate is independent on its past size. Thus, testing for Gibtrat's Law implicitly entails that firm's growth rate is defined as a random walk, i.e, we can write:

$$
y_{i t}=\gamma_{0}+\gamma_{1} y_{i t-1}+u_{i t}
$$

where $y_{i t}$ is the $\log$ size of firm $i$ at time $t$ and $u_{i t}$ is an i.i.d. error term. If $\gamma_{1}$ is equal to unity, Gibrat's law is verified, whereas if $\gamma_{1}<0$ small firms grow faster than the larger ones and we observe convergence 
toward the mean. If $\gamma_{1}>1$ large firms grow faster and the growth path is unstable.

The empirical literature presents results that are controversial as they crucially depend on the characteristics of the data sets used for the empirical investigation. In addition, the methodological development of the literature on panel unit root tests has contributed, on the one hand, to include such methods within the more general framework of the test for stationarity which were originally developed within the time series literature. On the other hand, this relatively new literature has increased the uncertainty about the outcome of the test, given the assumptions on which such tests are based and the controversy that this brings about.

One of the most significant limitations of the literature on testing for Gibrat's Law is that results crucially depends on the characteristics of the data set used for their implementation. Early studies have typically focused on cross-section data, thus lacking to take into consideration the real dynamics and evolution of firm size across time and industries. The application of these tests to different industries, although relevant for understanding dynamics related to specific conditions and technological opportunities, does not provide a general validity of the hypothesis being tested for. Also, the consideration of firms' birth and death rate is crucial to obtain estimates that otherwise would suffer of significant selection bias. Goddard et al. (2002) provide a detailed analysis of panel unit root tests for a set of Japanese manufacturing firms, 
concluding that firm size is mean reverting towards heterogeneous values,and thus Gibrat's Law should be rejected. Oliveira and Fortunato (2006b) extend the testing procedure by including firm and industry specific factors affecting size growth, suggesting that age, and financial constraints play a significant role. In particular firm age and leverage negatively affect size growth, while a liquidity index exerts a positive impact. Almus (2000) apply tests for Gibrat's Law to a set of young German manufacturing firms, rejecting the underlying hypothesis of proportionate growth rate.

Lotti et al. (2009) test the hypothesis for a set of Italian firms belonging to the radio, TV and communication equipment industries, by taking also account for possible selection bias related to firms' death and birth rate. They do not find support to the hypothesis being tested for. However, they suggest that convergence towards Gibrat's Law is verified, in that a separate test including only the final years of the period taken into consideration, allows for not rejecting the hypothesis. This fact is interpreted as an indication that market selection, at least in these industries, produces a final condition in which the dynamics of industrial business is coherent with Gibrat's pattern. Such a pattern is also verified by Fotopoulos and Giotopoulos (2010) within medium and large Greek manufacturing firms; however, Gibrat's Law is rejected for micro, small and young firms. 


\section{The data set and the stylised facts}

\subsection{General overview}

Our data set is represented by the Panel data on the balance sheets of corporations with employees (ISTAT), an integrated data source providing demographic and economic information on the population of Italian limited companies during the period 2001-2014. Firms' demographic information is based on the ASIA archive (Statistical Register of Active Businesses), which was set-up in 1996 and is regularly updated according to the European Council Regulations. The ASIA register covers all enterprises carrying on economic activities contributing to the formation of Italian gross domestic product and, thus, it is used by ISTAT for sample and census investigations. Our dataset also provides annual information about imports and exports and, thus, allows one to widening the analysis to firms' internationalization patterns. From the original dataset we select an unbalanced panel of almost 193 thousand manufacturing firms (more than one million three hundred thousand observations) during 14 years, which include the financial crisis of 2008 and its aftermath. Descriptive statistics are reported in Table 1.

This data set enables us to analyse transitions between size classes along the whole period. We thus consider long-run transitions, i.e. , upsizing and downsizing for those firms which were active in 2001 and 2014, in order to gather information on firm size dynamics and persistence patterns. 
Table 1: Variable definition and descriptive statistics - Selected years

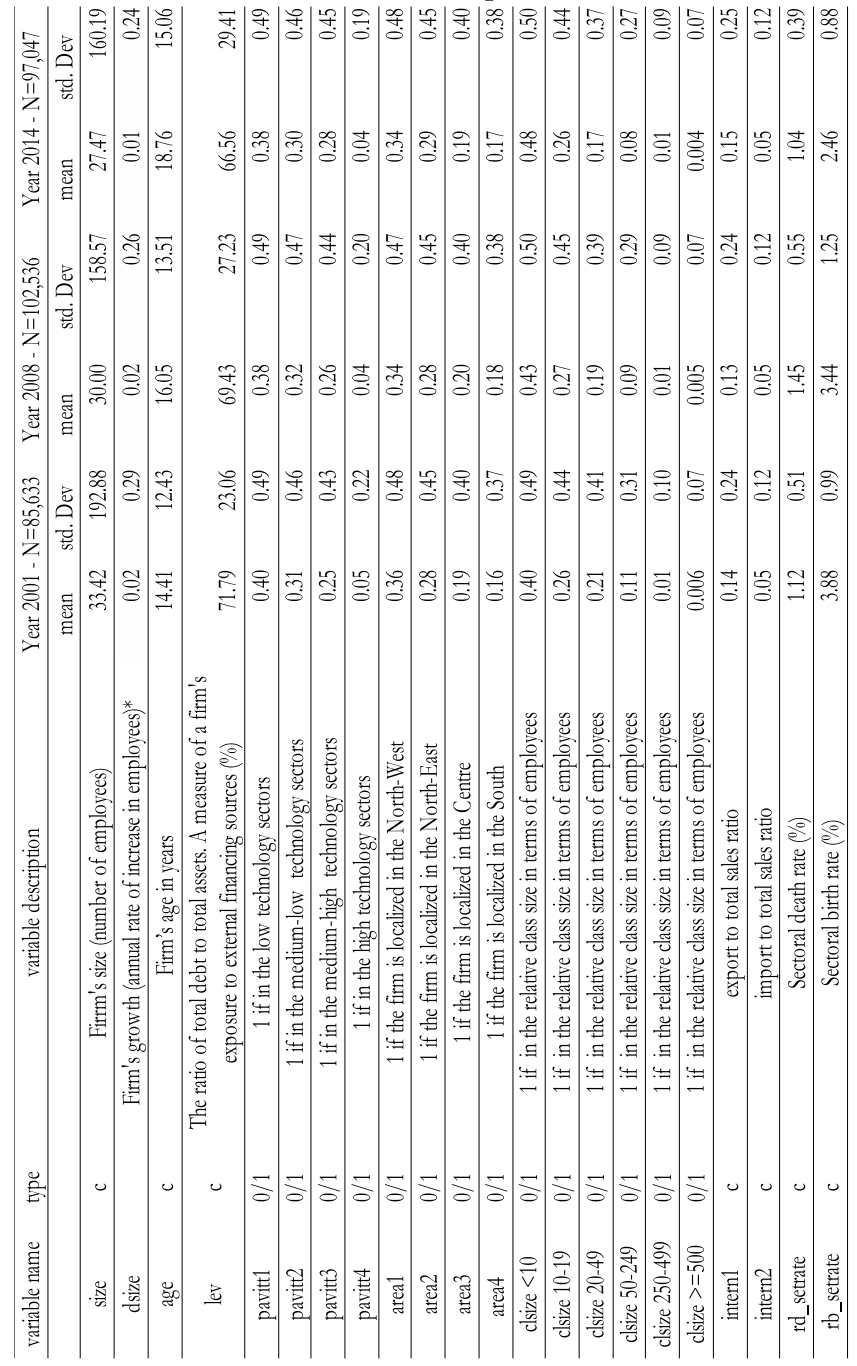


In particular, if one considers the 2001-2014 transition probability matrix (TPM), persistence is the dominant chracteristics as the persistence rate varies between almost $95 \%$ for larger firms and $84 \%$ for firms with 10 to 20 employees (Table 2).

We have also calculated at the bottom of the matrix the so-called steady-state distribution of firms by their size. The implied steadystate firm size distribution may be derived by imposing the steady-state condition, i.e., the outflows and inflows from one state counterbalance each other. In other words, this implies that:

$$
x A=x
$$

where $x$ is the row vector, which reflects the distribution of firms according to their size, and $A$ is the associated TPM. Equation (2) implies that the steady-state distribution may be found by calculating the eigenvector associated with the unit eigenvalue ${ }^{1}$. The implied long-run distribution indicates that almost $66 \%$ of firms belong to the bottom class and slightly more than $27 \%$ to the second, i.e., $94 \%$ of firms have fewer than 20 employees. Medium to large-sized enterprises represent the smallest minority of the population of Italian manufacturing firms.

This evidence is coherent with previous findings based on the results of the Industry Census (ISTAT, 2013), which shows that the bulk of Italian firms (80\%) belong to a conservative or traditional cluster and typically operate with a defensive strategy targeted to the maintenance

\footnotetext{
${ }^{1}$ For a more detailed description of the steady state definition, see Basu (2003).
} 
of the acquired market share.

On the other hand, it is suggested that a small minority of firms, representing approximately $5 \%$ of companies, have adopted more aggressive strategies devoted to increasing market share, which enables them to compete in international markets. Among this tiny group, even fewer firms show a large size, a complex organisation structure and a global strategy.

Table 2: Transition probabilities between size classes during the period 2001-2014 (yearly averages)

\begin{tabular}{lrrrrrrr}
\hline & & & & \multicolumn{3}{c}{500 and } \\
more & Total \\
\hline less than 10 & & & & & & & \\
10 & $\mathbf{9 1 . 8}$ & 7.6 & 0.6 & 0.1 & 0.0 & 0.0 & 100.0 \\
$10-19$ & 10.1 & $\mathbf{8 4 . 1}$ & 5.7 & 0.1 & 0.0 & 0.0 & 100.0 \\
$20-49$ & 1.0 & 7.7 & $\mathbf{8 8 . 2}$ & 3.1 & 0.0 & 0.0 & 100.0 \\
$50-249$ & 0.3 & 0.3 & 6.0 & $\mathbf{9 2 . 6}$ & 0.7 & 0.0 & 100.0 \\
$250-499$ & 0.0 & 0.0 & 0.1 & 8.7 & $\mathbf{8 8 . 6}$ & 2.5 & 100.0 \\
500 and more & 0.0 & 0.0 & 0.1 & 0.2 & 4.9 & $\mathbf{9 4 . 9}$ & 100.0 \\
Stcady-state distribution & 65.6 & 27.2 & 3.2 & 2.9 & 0.7 & 0.8 & \\
\hline
\end{tabular}

Notes. Variable size and age in log terms. $\mathrm{m} 2$ is asymptotically $\mathrm{N}(0,1)$ test for second order serial correlation of the residuals. P-values in parentheses.

\subsection{Firm size distribution}

In this section, we further analyse the characteristics of the data set, by testing whether firms size (as described by the number of employees) may be represented by a lognormal distribution. We describe the fit of the size distribution of firms to the data set previously described, i.e. the panel of manufacturing firms with balance sheet information, and we also apply the same procedure to a second data set, which represents the population of firms drawn from the ASIA archive. This second fit enables us to verify whether the selected panel presents distribu- 
tions that are consistent with those of the population of manufacturing firms ${ }^{2}$.

We then consider the firm size distributions, to test whether, according to the empirical literature, the LN model presents the best fit. The competing models are: gamma $(\mathrm{G})$, unimodal inverse Gaussian (IG), Weibull (W), and logistic (L) ${ }^{3}$.

To compare models with the same number of parameters, in terms of goodness-of-fit, we use the log-likelihood (in addition to the criteria described below). Comparison of models with differing number of parameters is accomplished, as usual, via the Akaike information criterion (AIC; Akaike, 1974) and the Bayesian information criterion (BIC; Schwarz, 1978) that, in our formulation, need to be maximized.

In Table 6 reported in the Appendix some descriptive statistics related to different years are displayed. Data results (for all the years) right-skewed and leptokurtic, with a long right-tail. To give a graphical idea about the distribution, we report in Figure 1 the histogram of the data for the year 2014 .

Figure 2 presents a model comparison in terms of goodness-of-fit. In particular, that figure reports, for each year and estimated model, the AIC value. It is easy to note as the ranking in terms of that index

\footnotetext{
${ }^{2}$ It is worth recalling that the selected panel results from merging balance sheet data and statistical information on employees derive from the ASIA archive.

${ }^{3}$ Parameters are estimated via the maximum likelihood (ML) procedure and the whole analysis is made in the $\mathrm{R}$ statistical software ( $\mathrm{R}$ Core Team, 2016). For the models LN, G, W, and L the estimated parameters are obtained by the fitdist() function of the fitdistrplus package (Delignette-Muller et al., 2017), while for the model IG the estimates are obtained using the function provided in Punzo et al. (2017)
} 
is the same for all the years. The same result holds if the BIC index is considered (for brevity's sake the results are not reported here). In particular, the best model results to be the LN. The second best model is the IG which produce AIC values always lower than the LN model (even if from the Figure 2 it seems that LN and IG provide the same AIC).

Moving to our data base of manufacturing firms with balance sheets, Table 7 reports some descriptive statistics ${ }^{4}$. The histogram of the observed data for year 2014 is reported in Figure 3. Similarly to the first dataset also here we observe right-skewness and leptokurtosis.

The comparison among fitted models is presented in Figure 4 where the AIC for each fitted model is reported along with the corresponding year. As can be easily noted, the ranking induced by the AIC is equal for all years, and results are similar for the BIC criteria. Also, one should note that the ranking corresponds to that obtained in the first data set. Moreover, as in the previous application, the first and the second best models are the LN and IG, respectively.

\section{Firms' growth: tests and further evidence}

According to the analysis previously described, it is confirmed that firm size is better described by a lognormal distribution. This result is

\footnotetext{
${ }^{4}$ Differences in terms of mean size are justified on the ground that the ASIA archive, compared to our data set, also includes small individual firms for which balance sheet information is not available from the Italian public register, thus our analysis excludes these firms.
} 
coherent with the stylised facts of firms size distribution detected in the international literature. Given this preliminary inspection of the data, we formally test for the validity of Gibrat's Law. This latter implies, according to equation (1) that firm size dynamics may be described by a random walk. Thus, a first step is represented by testing for unit root the time path of firm size (equation 1). We are aware of the limitations that these tests bring about, particularly when they are applied to a panel framework. However, keeping this consideration into account, we consider such tests as preliminary and complementary to the more general specification of firms growth rate. We thus present panel unit root tests in Table 3 .

According to the results of these tests we reject the unit root hypothesis and, therefore, the validity of Gibrat's Law ${ }^{5}$.

Given this preliminary analysis, we decided to model a firms' growth rate also taking into account specific characteristics which may crucially affect a firm's growth rate. In particular, we consider:

- the degree of internationalisation expressed regarding the relative value of import and exports with respect to sales,

- financial efficiency, i.e. leverage, the debt-equity ratio,

- age.

In addition, we control for sectoral birth and death rate, thus en-

\footnotetext{
${ }^{5} \mathrm{We}$ are aware of the fact that these tests are subject to limitations because of the underlying assumptions. However, they provide a first step in the analysis of the validity of Gibrat's Law.
} 
abling our estimates to take into consideration that some firms exit and other enter the market during the sample period. However, one has to consider that exit rates are relatively low even during the great recession of 2008-09, due to institutional and legislative factors that have been introduced over the years and in particular in the aftermath of the financial crisis.

We, therefore, estimate the following equation:

$$
\begin{aligned}
& \Delta \text { size }_{i t}=\beta_{0}+\beta_{1} \Delta \text { size }_{i t-1}+\beta_{2} \Delta \text { size }_{i t-2}+\beta_{3} \text { size }_{i t-1} \\
& +\beta_{4} i n t e r n 1_{i t}+\beta_{5} i n t e r n 2_{i t}+\beta_{6} a g e_{i t}+\beta_{7} l e v_{-} p c_{i t} \\
& +\beta_{8} r d \_s e t r a t e_{t}+\beta_{9} r b \_s e t r a t e_{t}+\epsilon_{i t}
\end{aligned}
$$

where $\epsilon_{i t}$ is a normal distributed i.i.d. error component and $i$ and $t$ identify respectively firms and time.

\begin{tabular}{lrr}
\multicolumn{3}{c}{ Table 3: Unit Root Tests } \\
\hline Choi tests & statistic & p-value \\
\hline Inverse chi-squared & 87,600 & 0.0000 \\
Inverse normal & -7.0131 & 0.0000 \\
Inverse logit t & -27.0264 & 0.0000 \\
Modified inverse chi-squared & 86.8466 & 0.0000 \\
& & \\
\hline Levin-Lin-Chu test & statistic & p-value \\
\hline Adjusted t* & -1300 & 0.0000 \\
& \multicolumn{2}{c}{} \\
Number of panels & 29,020 \\
Number of periods & 14 \\
\hline
\end{tabular}

Notes. Unit root tests according to Choi (2001) and Levin, Lin, Chu (2002). We consider a panel data model with the following autoregressive component: $\Delta y_{i t}=\gamma_{1} y_{i t-1}+k^{\prime} \delta_{i}+\gamma_{2} \Delta y_{i t-2}+u_{i t}$, where $k^{\prime} \delta_{i}$ are panel specific means. H0: all panels contain uit roots H1: At least one panel is stationary.

As concerns the explanatory variables, size is the log of firm size, 
intern 1 and intern 2 are respectively the value of a firm's exports and imports to total sales, age is the log value of a firm's age, lev_pc is a leverage index, i.e. the total debt to equity ratio, and $r d \_s e t r a t e$ and rb_setrate represent sectoral death and birth rates respectively.

Thus, we estimate an augmented firm size growth equation, in that together with the traditional specification which includes a lagged term and difference terms of the size variable, we control for other factors which are typically recognised as crucial factors affecting a firm's ability to grow according to the international empirical literature (Oliveira and Fortunato, 2006b,a).

Table 4: Firms' growth: dynamic panel data estimation (ArellanoBond). Period 2001-2014

\begin{tabular}{|c|c|c|c|c|c|c|c|}
\hline \multirow[t]{2}{*}{ Variable } & \multirow{2}{*}{$\begin{array}{c}\text { full } \\
\text { sample }\end{array}$} & \multicolumn{6}{|c|}{ by size class } \\
\hline & & less than 10 & $10-19$ & $20-49$ & $50-249$ & $250-499$ & 500 and more \\
\hline \multirow[t]{2}{*}{$\begin{array}{l}\text { L.dsize } \\
\end{array}$} & $-0.0272 * * *$ & $0.0135^{* *}$ & $0.0263^{* * *}$ & $-0.0193^{* * *}$ & $-0.0745^{* * *}$ & $-0.0563^{*}$ & $-0.0245 *$ \\
\hline & {$[0.00338]$} & {$[0.00542]$} & {$[0.00502]$} & {$[0.00673]$} & {$[0.0126]$} & {$[0.0336]$} & {$[0.0126]$} \\
\hline \multirow[t]{2}{*}{ L2.dsize } & $-0.0249 * * *$ & -0.00204 & 0.00356 & $-0.0137^{* * * *}$ & $-0.0498^{* * * *}$ & -0.0191 & -0.00327 \\
\hline & {$[0,00217]$} & {$[0.00359]$} & {$[0.00306]$} & {$[0.00418]$} & {$[0.00601]$} & {$[0.0146]$} & {$[0.0174]$} \\
\hline \multirow[t]{2}{*}{ L.size } & $-0.898^{* * * *}$ & $-0.913^{* * *}$ & $-1.014 * * *$ & $-0.941 * * *$ & $-0.874 * * *$ & $-0.904^{* * * *}$ & $-0.944^{* * * *}$ \\
\hline & {$[0.00487]$} & {$[0.00726]$} & {$[0.00838]$} & {$[0.0105]$} & {$[0.0179]$} & {$[0.0373]$} & [0.0928] \\
\hline \multirow[t]{2}{*}{ intern1 } & $0.104^{* * *}$ & $0.144^{* * *}$ & $0.0800^{* * *}$ & $0.0663^{* * *}$ & $0.0805^{* * *}$ & 0.0941 & 0.0607 \\
\hline & {$[0.00494]$} & {$[0.0106]$} & {$[0.00706]$} & {$[0.00690]$} & {$[0.0126]$} & {$[0.0628]$} & {$[0.0783]$} \\
\hline \multirow[t]{2}{*}{ intern2 } & $0.0250 * * *$ & $0.0415^{* *}$ & 0.0218 & 0.0141 & 0.0233 & -0.0153 & -0.0794 \\
\hline & {$[0.00924]$} & {$[0.0205]$} & {$[0.0145]$} & {$[0.0122]$} & {$[0.0185]$} & {$[0.0436]$} & {$[0.147]$} \\
\hline \multirow[t]{2}{*}{ age } & $-0.116^{* * *}$ & $-0.321^{* * *}$ & $0.0830^{* * *}$ & $0.158^{* * *}$ & $0.161 * * *$ & $0.121 * *$ & 0.046 \\
\hline & {$[0.00337]$} & {$[0.00477]$} & {$[0.00538]$} & {$[0.00825]$} & {$[0.0166]$} & {$[0.0527]$} & {$[0.0546]$} \\
\hline \multirow[t]{2}{*}{ lev_pc } & $-0.00141^{* * *}$ & $-0.00199 * * *$ & $-0.00107^{* * *}$ & $-0.000653^{* * *}$ & $-0.000428^{* * *}$ & -0.0000129 & $0.000790^{*}$ \\
\hline & {$[3.65 \mathrm{e}-05]$} & {$[6.20 \mathrm{e}-05]$} & [5.56e-05] & {$[6.08 \mathrm{e}-05]$} & {$[8.83 \mathrm{e}-05]$} & {$[0.000463]$} & {$[0.000417]$} \\
\hline \multirow[t]{2}{*}{ rd_setrate } & $0.00812^{* * *}$ & $0.00760^{* * * *}$ & $0.00744 * * *$ & $0.0113^{* * *}$ & $0.0122^{* * * *}$ & 0.0101 & -0.0024 \\
\hline & {$[0.000714]$} & {$[0.00141]$} & {$[0.00102]$} & {$[0.00110]$} & {$[0.00158]$} & {$[0.00652]$} & {$[0.00826]$} \\
\hline \multirow[t]{2}{*}{ rb_setrate } & $0.00805^{* * *}$ & $0.00772 * * *$ & $0.00787 * * *$ & $0.00867^{* * * *}$ & $0.00692 * * *$ & 0.00593 & -0.00752 \\
\hline & {$[0.000497]$} & {$[0.000941]$} & {$[0.000699]$} & {$[0.000787]$} & {$[0.00133]$} & {$[0.00659]$} & {$[0.00468]$} \\
\hline \multirow[t]{2}{*}{ Constant } & $2.858^{* * * *}$ & $2.561^{* * * *}$ & $2.518^{* * * *}$ & $2.753 * * *$ & $3.391 * * * *$ & $4.816^{* * *}$ & $6.313^{* * * *}$ \\
\hline & [0.0144] & {$[0.0182]$} & [0.0196] & {$[0.0298]$} & {$[0.0651]$} & {$[0.196]$} & {$[0.611]$} \\
\hline Obs. & 634,196 & 224,522 & 186,194 & 141,338 & 72,055 & 6,276 & 3,811 \\
\hline n. of firms & 110,333 & 57,165 & 44,160 & 28,767 & 12,793 & 1,297 & 632 \\
\hline $\mathrm{m} 2$ & $-0.05(0.96)$ & $1.44(0.15)$ & $-1.01(0.31)$ & $2.08(0.03)$ & $0.93(0.34)$ & $-1.42(0.15)$ & $-0.51(0.60)$ \\
\hline
\end{tabular}

Notes. Variable size and age in log terms. $\mathrm{m} 2$ is asymtotically $\mathrm{N}(0,1)$ test for second order serial correlation of the residuals. P-values in parentheses.

The proposed specification confirm the previous results which reject 
Gibrat's Law and suggest that it is crucial to consider other determinants of firm's growth rate which enables us to evaluate more precisely the role of small and medium-sized companies ${ }^{6}$. Indeed, the impact of size on the growth rate is not linear, in that it tends to increase as the size class increases until the "less than 50 employees" size. Such an impact decreases for companies in the 50-249 class, and then it increases for the remaining upper classes.

The impact of age is differentiated as well, in that although a negative effect is observed for the whole sample, it is however positive for all size classes but the first, i.e. firms with les than 10 employees. It is also worth noting that the impact is increasing until the penultimate class, while it is not significant for large businesses (500 employees and more).

This is coherent with the fact that, on the one hand, small firms do experience higher birth and dead rates, and on the other largest companies have encountered non negligible restructuring over the whole period of analysis. This phenomenon involved all companies independently on their age.

This finding complements previous results that underline how the distribution of firms size becomes thicker on the right tail; Cabral and Mata (2003) and Cirillo (2010) analyse the statistical properties of the size distribution of Portuguese and Italian firms respectively, by focusing on the impact of age.

\footnotetext{
${ }^{6}$ Recall that, in order to reject Gibrat's Law, the coefficient on the lagged size variable should not be significantly different from zero.
} 
Firms' financial condition, as described by the leverage variable, has a negative effect on firm's growth. The impact is however mild and becomes negligible as firm size increases.

The ability to sell product on international markets is captured by the variable that represents the ratio of exports to total sales (intern 1 ). It's impact is positive and driven by the effect that it exerts within the group of small firms. This result is important as it highlights that exports and, therefore, the ability to enter international markets may be a driver of a firm's growth.

This is particularly true for small business, although entering international markets also entails possible increases in costs not always compensated by a corresponding increase in revenues ${ }^{7}$.

We have also considered the ratio of the value of imports to total sales (intern2), to indicate another source of openness. The impact is less relevant, as expected, compared with that of exports and is confined only to very small companies.

We control for sectoral birth and death rates. It is worth recalling that these variables are relatively steady, particularly the death rate variable which reflects the negative impact of new legislation introduced in the aftermath of the financial crisis.

The adopted specification of the firms' growth equation includes two lagged values of the growth rate, to tackle possible autocorrelation

\footnotetext{
${ }^{7}$ In a previous study on Italian manufacturing businesses Bartoloni and Baussola (2017) find that the impact of exporting in international markets on firms' operating profits is either negligible or slightly negative, thus suggesting that their could be external diseconomies in accessing foreign markets, particularly for small firms.
} 
that may, therefore, affect the estimates. On the whole, this dynamic specification provides results that avoid this problem as suggested by the autocorrelation tests.

In general, we observe that firms' size dynamics does not show a persistence pattern. However, this fact does not rule out the inability of firms to pass over another size class, thus providing persistency within a given reference size class. In other words, we observe that size dynamics is confined within the border of the reference size, thus causing the aggregate effect that we have previously underlined, i.e., the inability to significantly increase the number of medium-large sized companies.

For this reason, we decided to specify a logit model, which express the probability that a firm increases its size relatively to the average size of its class ${ }^{8}$. This issue is presented and discussed in the next section.

\section{Long-run transitions}

Given the previous discussion, we specify a logit model in which the probability of increasing size depends on a set of sectoral, geographical and firm-specific variables, as we used in the previous specifications of firms' growth rate. Equation (4) summarised the model:

\footnotetext{
${ }^{8}$ We have also experimented a different long-run specification in which the upsizing probability has been defined with respect to a change in the reference size class. However, given the few changes that occur the model cannot be estimated significantly.
} 


$$
z_{i}=\theta_{0}+\sum_{i=1}^{N} \theta_{j} x_{i j}+\mu_{i}
$$

where $z_{i}$ is a dummy variable taking the value of one if firm $i$ has increased its size, and zero otherwise, $\theta_{0}$ is a constant, $\theta_{j}$ is the coefficient of explanatory variable $x_{i j}$ and $\mu_{i}$ is the error term whose cumulative distribution is assumed to be logistic.

We show in Table 4 results for the logistic model. The export propensity is positive and significant and implies that a unit increase (percentage point) in the export to sales ratio brings about an $8.1 \%$ increase in the upsizing probability.

The impact of age is significant, and its impact implies that a unit increase in age (expressed in log) increases the upsizing probability by more than $5 \%$. This result may appear contradictory, given the previous findings of Table 4 where the impact of age is negative.

It is worth noting that the logistic regression presents long-run estimate of the probability of increasing a firm's size compared to the average size of the corresponding class. Thus, it could be the case that in a year by year regression and considering just the marginal impact of age on size, the effect could result negative. Also, one should note that interacting the first size class $(<10$ employees $)$ with the age variable, determine a negative effect, thus confirming the result derived in the growth regressions of Table 4.

A firms' financial condition negatively affects the upsizing probability, although the impact is very mild. Industry characteristics, sum- 
Table 5: Upsizing probability 2001-2014 - Logistic estimation (Odds Ratios)

\begin{tabular}{|c|c|c|c|}
\hline Variable & $\begin{array}{c}\text { Model } \\
(1)\end{array}$ & $\begin{array}{c}\text { Model } \\
\text { (2) }\end{array}$ & $\begin{array}{c}\text { Model } \\
\text { (3) }\end{array}$ \\
\hline \multirow[t]{2}{*}{ intern1 } & $1.081^{*}$ & $1.089 * *$ & $1.738^{* * *}$ \\
\hline & [0.0459] & {$[0.0461]$} & {$[0.0808]$} \\
\hline \multirow[t]{2}{*}{ age } & $1.054 *$ & $1.054^{*}$ & $1.378^{* * *}$ \\
\hline & {$[0.0313]$} & {$[0.0313]$} & {$[0.0505]$} \\
\hline \multirow[t]{2}{*}{ lev_pc } & $0.999 * * *$ & $0.999 * * *$ & $0.999 * * *$ \\
\hline & {$[0.000429]$} & [0.000429] & [0.000435] \\
\hline \multirow[t]{2}{*}{ area1 } & 1.069 & 1.074 & 1.027 \\
\hline & {$[0.0487]$} & [0.0489] & {$[0.0475]$} \\
\hline \multirow[t]{2}{*}{ area2 } & $1.093^{*}$ & $1.095^{* *}$ & $1.104^{* *}$ \\
\hline & {$[0.0505]$} & {$[0.0506]$} & [0.0518] \\
\hline \multirow[t]{2}{*}{ area3 } & $1.146^{* * *}$ & $1.152^{* * *}$ & $1.092^{*}$ \\
\hline & {$[0.0572]$} & {$[0.0577]$} & {$[0.0554]$} \\
\hline \multirow[t]{2}{*}{ clsize $<10$} & & & $12.64 * * *$ \\
\hline & & & [3.519] \\
\hline \multirow[t]{2}{*}{ clsize $10-19$} & & & $6.372^{* * *}$ \\
\hline & & & [1.039] \\
\hline \multirow[t]{2}{*}{ clsize $20-49$} & & & $4.453 * * *$ \\
\hline & & & {$[0.723]$} \\
\hline \multirow[t]{2}{*}{ clsize $50-249$} & & & $2.404 * * *$ \\
\hline & & & {$[0.392]$} \\
\hline \multirow[t]{2}{*}{ clsize $250-499$} & & & $3.233^{* * *}$ \\
\hline & & & {$[0.618]$} \\
\hline \multirow[t]{2}{*}{ clsize $<10^{*}$ age } & & & $0.846^{* *}$ \\
\hline & & & {$[0.0575]$} \\
\hline \multirow[t]{2}{*}{ pavitt2 } & $1.061^{* *}$ & & $1.097^{* * *}$ \\
\hline & {$[0.0308]$} & & {$[0.0323]$} \\
\hline \multirow[t]{2}{*}{ pavitt3 } & 1.033 & & 1.045 \\
\hline & [0.0309] & & {$[0.0318]$} \\
\hline \multirow[t]{2}{*}{ pavitt4 } & 1.075 & & $1.186^{* * *}$ \\
\hline & {$[0.0679]$} & & {$[0.0766]$} \\
\hline \multirow[t]{2}{*}{ mrb_setrate } & & 1.022 & \\
\hline & & {$[0.0175]$} & \\
\hline \multirow[t]{2}{*}{ mrd_setrate } & & $0.896^{* * *}$ & \\
\hline & & {$[0.0363]$} & \\
\hline \multirow[t]{2}{*}{ Constant } & 0.841 & 0.904 & $0.0612^{* * *}$ \\
\hline & {$[0.0930]$} & {$[0.107]$} & {$[0.0132]$} \\
\hline LR chi2 & $32.84(8)$ & $35.44(8)$ & $974.37(15)$ \\
\hline Observations & 29,409 & 29,409 & 29,409 \\
\hline
\end{tabular}

Notes. Variable age in log. Firms which are present at the beginning and at the end of the period. Probability of increasing firms' size above their respective class size averaged over the entire period. 
marised by the Pavitt technological dummy variables, suggest that as technological level increases the upsizing probability increases accordingly with an impact that varies between $3.3 \%$ and $7.7 \%$ with respect to the lowest technological level.

Death and birth rates are also included in a separate logistic regression which shows that only the former is significant and reduces the probability of increasing a firm's size by more than $10 \%$.

This analysis is therefore complementary to the previous panel investigation on firms' growth rate. It focuses on evaluating the extent to which firm and industry characteristics affect upsizing in the long run. The ability to sell on international market and industry technological opportunities play a crucial role in this context. However, this positive impact, although significant and not negligible, is still insufficient to determine a right shift of the size distribution of firms implying that the weight of medium size enterprises is increased.

\section{Conclusions}

It is a well established fact that the ability of firms to compete internationally crucially depends on their ability to innovate and to introduce new products or processes into the market. This ability requires a more structured and complex business organisation, and ultimately a firm size that is suited to the size of the market.

We have carried out an empirical investigation concentrating on a 
large sample of Italian manufacturing firms over the last 14 years.

We first analysed the statistical properties of firm size distribution and then calculated a Transition Probability Matrix that enabled us to quantify movements between size classes and thus determine transition probabilities over the long run.

Results suggest that downsizing, i.e. a significant reduction in employees in large companies, is a prevailing tendency. There are, however, weak signs suggesting that a small number of medium-sized firms have marginally increased in size and so also in their ability to compete in the global market.

We therefore further investigated the relationship between the rate of growth in a firm's size and its past size level, conditional on specific firm and industry characteristics. This investigation followed a preliminary test, including panel unit root tests, for the validity of Gibrat's Law, which was rejected.

The panel growth regressions implicitly confirm previous unit root tests and suggest that small firms tend to grow faster than larger firms. This evidence is also confirmed by regressions for the sample of firms belonging to the reference class size.

Export intensity plays a significant role in affecting the size growth rate; the latter is also positively affected by industry characteristics related to technological levels.

We have also taken into account possible bias related to business demography by controlling for industry death and birth rates. 
Although we estimated significant negative effects between growth and size, the impact observed in the long run does not lead to significant modification of the size distribution of firms. In other words, small- and medium-sized firms fail to climb the ladder, i.e. they fail to pass into higher size classes.

This evidence is further confirmed using a logit model which enabled us to specify a firm's upsizing probability between the initial and final dates of the sample period.

Thus significant questions arise, given that the inability of small and medium sized firms to effectively increase in size may negatively affect their ability to compete in international markets which are often characterised by new technological paradigms. 


\section{Appendix}

Table 6: ASIA: descriptive statistics for years 2000-2014

\begin{tabular}{ccccccc}
\hline Year & No. Of Obs. & Mean & St. Dev & Skewness & Kurtosis & Maximum \\
\hline 2000 & 308402 & 13.899 & 75.205 & 53.370 & 4227.044 & 8782 \\
2001 & 295910 & 14.082 & 75.524 & 54.209 & 4463.026 & 9496 \\
2002 & 276854 & 14.275 & 74.886 & 54.362 & 4643.302 & 9778 \\
2003 & 260153 & 14.601 & 71.168 & 47.443 & 3615.791 & 9202 \\
2004 & 243234 & 14.823 & 70.831 & 45.268 & 3307.297 & 9000 \\
2005 & 229776 & 15.045 & 71.978 & 44.088 & 3102.821 & 8938 \\
2006 & 215478 & 15.437 & 73.879 & 43.944 & 3055.735 & 8872 \\
2007 & 203225 & 16.067 & 80.363 & 48.040 & 3703.838 & 9941 \\
2008 & 193112 & 16.445 & 83.000 & 45.883 & 3204.826 & 8929 \\
2009 & 181637 & 16.453 & 83.080 & 44.725 & 3049.702 & 8741 \\
2010 & 172987 & 16.469 & 83.795 & 43.785 & 2922.995 & 8490 \\
2011 & 167292 & 16.460 & 83.139 & 43.826 & 2964.425 & 8518 \\
2012 & 159558 & 16.632 & 82.770 & 42.741 & 2862.302 & 8682 \\
2013 & 152694 & 16.712 & 83.531 & 41.832 & 2749.886 & 8820 \\
2014 & 144123 & 17.050 & 85.023 & 42.411 & 2904.879 & 9414 \\
\hline
\end{tabular}

Table 7: Panel Data on balance sheets: descriptive statistics for years 2001-2014

\begin{tabular}{ccccccc}
\hline Year & No. Of Obs. & Mean & St. Dev & Skewness & Kurtosis & Maximum \\
\hline 2001 & 85663 & 35.424 & 192.885 & 76.637 & 10268.717 & 32307 \\
2002 & 87643 & 34.276 & 179.653 & 71.339 & 9079.383 & 29147 \\
2003 & 85881 & 33.965 & 173.537 & 66.070 & 7360.544 & 25592 \\
2004 & 92091 & 33.093 & 166.789 & 62.126 & 6529.436 & 23641 \\
2005 & 94154 & 33.123 & 166.199 & 60.281 & 6096.152 & 22716 \\
2006 & 99006 & 32.178 & 162.553 & 60.663 & 6194.863 & 22741 \\
2007 & 100595 & 32.051 & 160.595 & 62.278 & 6511.053 & 22998 \\
2008 & 102536 & 31.996 & 158.571 & 60.324 & 6246.616 & 22641 \\
2009 & 100722 & 31.414 & 158.618 & 61.914 & 6622.398 & 23381 \\
2010 & 99847 & 30.467 & 158.130 & 67.897 & 8083.631 & 25179 \\
2011 & 100747 & 30.156 & 153.195 & 66.130 & 7908.020 & 24428 \\
2012 & 98941 & 29.529 & 137.546 & 63.362 & 7549.183 & 21846 \\
2013 & 97419 & 30.033 & 159.048 & 88.091 & 14034.687 & 30142 \\
2014 & 97047 & 29.465 & 160.186 & 96.927 & 16466.928 & 31654 \\
\hline
\end{tabular}




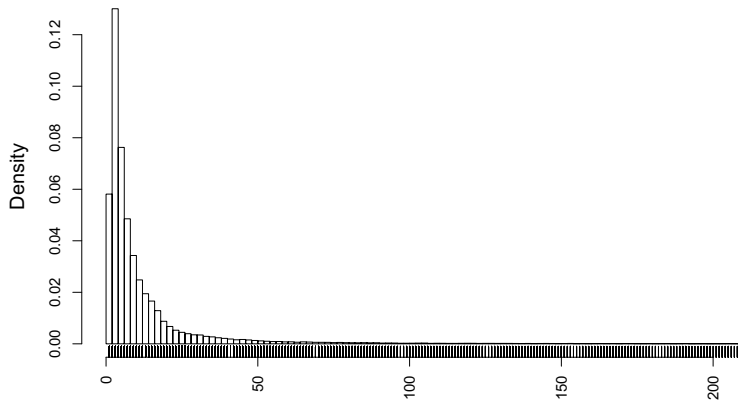

Figure 1: ASIA: histogram, year 2014.

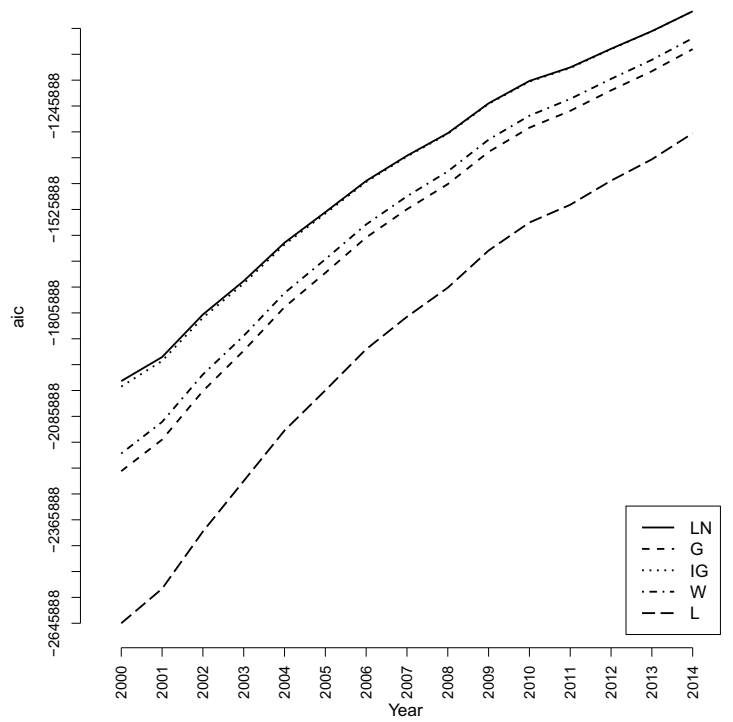

Figure 2: ASIA: AIC of fitted models for years 2000-2014. 


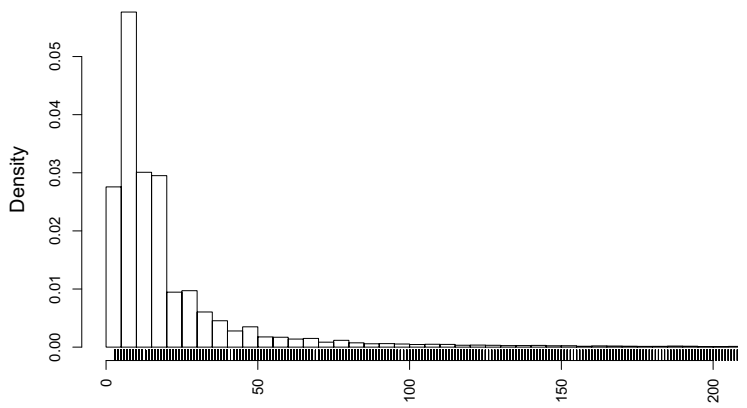

Figure 3: Panel Data on balance sheets: histogram, year 2014.

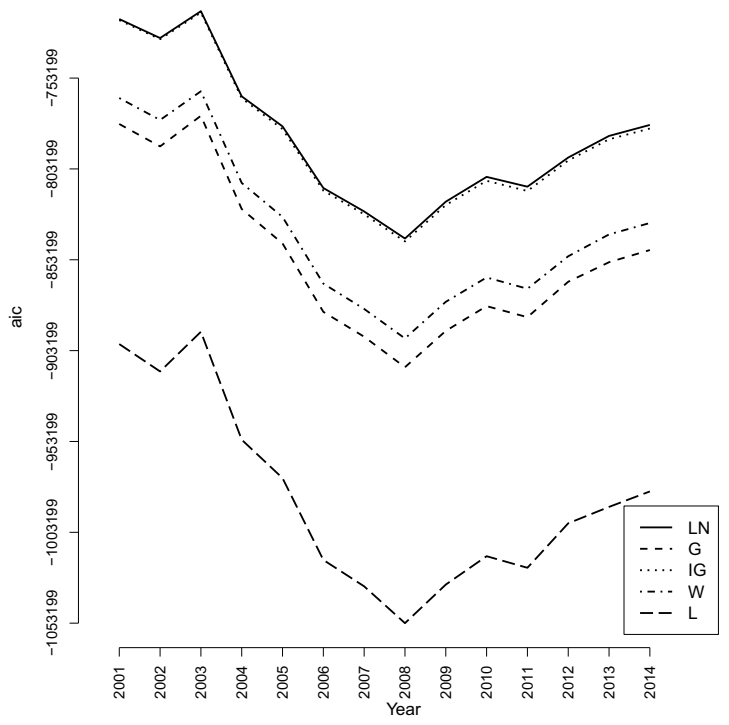

Figure 4: Panel Data on balance sheets: AIC of fitted models for years 2001-2014. 


\section{References}

Akaike, H. (1974). A new look at the statistical model identification. IEEE Transactions on Automatic Control, 19(6), 716-723.

Almus, M. (2000). Testing" gibrat's law" for young firms-empirical results for west germany. Small Business Economics, 15(1), 1-12.

Bartoloni, E. and Baussola, M. (2017). Driving business performance: innovation complementarities and persistence patterns. Industry and Innovation, pages 121.

Basu, A. K. (2003). Introduction to stochastic process. Alpha Science Int'l Ltd.

Cabral, L. and Mata, J. (2003). On the evolution of the firm size distribution: Facts and theory. American economic review, 93(4), 1075-1090.

Chen, J.-R. and Lu, W.-C. (2003). Panel unit root tests of firm size and its growth. Applied Economics Letters, 10(6), 343-345.

Choi, I. (2001). Unit root tests for panel data. Journal of international money and Finance, 20(2), 249-272.

Cirillo, P. (2010). An analysis of the size distribution of italian firms by age. Physica A: Statistical Mechanics and its Applications, 389(3), 459-466.

Delignette-Muller, M. L., Dutang, C., and Siberchicot, A. (2017). fitdistrplus: Help to Fit of a Parametric Distribution to Non-Censored or Censored Data. Version 1.0-8 (2017-02-01).

Fotopoulos, G. and Giotopoulos, I. (2010). Gibrat's law and persistence of growth in greek manufacturing. Small Business Economics, 35(2), 191-202.

Goddard, J., Wilson, J., and Blandon, P. (2002). Panel tests of gibrat's law for japanese manufacturing. International Journal of Industrial Organization, 20(3), 415-433. 
Im, K. S., Pesaran, M. H., and Shin, Y. (2003). Testing for unit roots in heterogeneous panels. Journal of econometrics, 115(1), 53-74.

ISTAT (2013). Istat rapporto annuale 2013.

Levin, A., Lin, C.-F., and Chu, C.-S. J. (2002). Unit root tests in panel data: asymptotic and finite-sample properties. Journal of econometrics, 108(1), 1-24.

Lotti, F., Santarelli, E., and Vivarelli, M. (2009). Defending gibrat's law as a longrun regularity. Small Business Economics, 32(1), 31-44.

Oliveira, B. and Fortunato, A. (2006a). Firm growth and liquidity constraints: A dynamic analysis. Small Business Economics, 27(2-3), 139-156.

Oliveira, B. and Fortunato, A. (2006b). Testing gibrat's law: empirical evidence from a panel of portuguese manufacturing firms. International Journal of the Economics of Business, 13(1), 65-81.

Punzo, A., Bagnato, L., and Maruotti, A. (2017). Compound unimodal distributions for insurance losses. Insurance: Mathematics and Economics.

R Core Team (2016). R: A Language and Environment for Statistical Computing. R Foundation for Statistical Computing, Vienna, Austria.

Schwarz, G. (1978). Estimating the dimension of a model. The Annals of Statistics, 6(2), 461-464. 
Printed by

Gi\&Gi srl - Triuggio (MB)

March 2018 
